\title{
Systemic RNAi in Caenorhabditis elegans
}

\author{
C.P. Hunter, W.M. Winston, C. Molodowitch, E.H. Feinberg, J. Shih, \\ M. Sutherlin, A.J. Wright, AND M.C. FitzGerald \\ Department of Molecular and Cellular Biology, Harvard University, Cambridge, Massachusetts 02138
}

\begin{abstract}
RNA interference (RNAi) in Caenorhabditis elegans induced by ingestion or injection of double-stranded RNA (dsRNA) spreads throughout the organism and is even transmitted to the progeny. We have identified two proteins required for spreading of RNAi, SID-1 and SID-2, whose structure, subcellular localization, and expression pattern have been informative for how dsRNA can be transported into and between cells. SID-1 is a transmembrane protein that functions as a pore or channel that transports dsRNA into and out of cells. Proteins homologous to SID-1 are present in a wide range of invertebrate and vertebrate animals but are absent from plants. SID-2 is a small transmembrane protein that is expressed in the gut and localizes strongly to the luminal membrane where it appears to act as a receptor for uptake of dsRNA from the environment. Characterization of SID-2 activity in a variety of Caenorhabditis nematodes indicates that $C$. elegans SID-2 may have a novel activity.
\end{abstract}

The initial report that dsRNA triggers posttranscriptional gene silencing in C. elegans was accompanied by the observation that identical phenotypes were elicited regardless of the site of dsRNA injection (Fire et al. 1998). This result implies that dsRNA or a genespecific silencing signal derived from the injected dsRNA is transported between cells and tissues (systemic RNAi). Similar observations of systemic gene silencing had been described in plants, where acquired viral resistance and transgene silencing information spread between old and new tissue and can even be transmitted across scions grafted onto the host plant (Baulcombe 2004). Building on the observation of systemic RNAi in C. elegans, two reports were published showing that RNAi could be triggered by exposure to environmental sources of dsRNA, either by soaking animals in concentrated solutions of dsRNA or by feeding animals Escherichia coli engineered to express gene-specific dsRNA (Tabara et al. 1998; Timmons and Fire 1998). How and why dsRNA or gene-specific signals are transported into animals and between cells was unknown.

\section{THE SCREEN}

To isolate mutations that specifically disrupt systemic RNAi, we designed a green fluorescent protein (GFP) transgene-based screen that monitored autonomous and systemic RNAi, separately (Winston et al. 2002). To monitor autonomous RNAi, we used a pharyngeal muscle promoter to express both GFP and a $g f p$ hairpin construct (Fig. 1). To monitor systemic RNAi, we expressed a nuclear localized GFP in all the body wall muscle cells. The pharyngeal GFP hairpin efficiently reduced GFP expression in the pharynx in all animals and reproducibly reduced nuclear localized GFP in anterior but not posterior body wall muscle cells, consistent with a pharynxderived gradient of silencing signal. Systemic silencing does not require coexpression of GFP in the pharynx, indicating that the systemic silencing signal is produced independently of the target mRNA, although the hairpin construct may serve that role. Mutations in genes such as rde-1 eliminated silencing of GFP in pharyngeal and body wall muscle cells, showing that the local and systemic RNAi observed in this strain require the same core RNAi machinery. Tabara et al. (1999) showed that $r d e-1$ function is not required to produce or transmit a systemic signal.

To identify systemic RNAi-defective (Sid) mutants, this triply transgenic strain was grown on bacteria that expressed a $g f p$ hairpin RNA, which effectively silenced GFP in all body wall muscle cells. We then isolated 200 candidate Sid mutants that reexpressed GFP in all body wall muscle cells but not in the pharyngeal muscle. Thus, these mutants were either defective for uptake of dsRNA from the bacteria and/or spreading of RNAi silencing from the pharynx to the body wall muscle cells. These 200 alleles identified three large complementation groups (20-100 alleles each) and several smaller uncharacterized complementation groups. sid-1(V), sid-2(III), and sid$3(X)$ were mapped, and sid-1 and sid-2 cloned by DNA transformation rescue (Winston et al. 2002 and in prep.). sid- 1 and likely sid-2 alleles were also isolated in independent screens (Timmons et al. 2003; Tijsterman et al. 2004).

\section{SID-1}

The predicted SID-1 protein encodes 11 potential transmembrane (TM) domains and a large extracellular amino-terminal domain (Fig. 2A). Experiments to determine the topology of individual TM domains confirmed predicted TM domains 1-4 and 6; however, the orientation of TM4 and TM6 indicates that TM5 cannot pass through the bilayer and requires that at least one additional predicted TM (7-11) also cannot pass through the bilayer (Feinberg and Hunter 2003). Thus, SID-1 contains 5,7 , or 9 TM domains. 

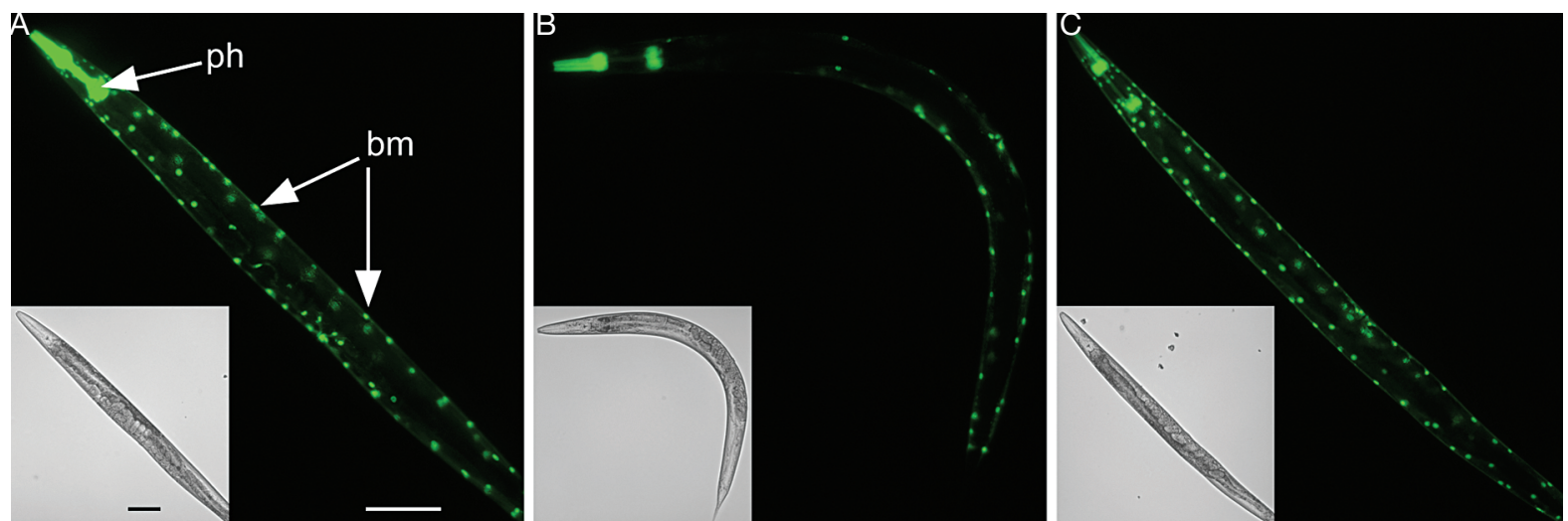

Figure 1. Direct visualization of systemic RNAi. $(A)$ Two separate transgenes direct GFP expression in pharynx and body wall muscle. $(B)$ The addition of a third transgene that expresses a $g f p$ hairpin to produce dsRNA in the pharynx silences the GFP reporter in the pharynx (autonomous RNAi) and silences GFP in anterior body wall muscles (systemic RNAi). (C) A sid-1 mutation in the background of the transgenes described for panel $B$. (ph) Pharynx muscle; (bm) body wall muscle. Insets are differential interference contrast images. (Reprinted in part, with permission, from Winston et al. 2002 [@ AAAS].)

GFP driven by 700 bp of sid-1 upstream sequences is detected in embryos and all larval stages. In adults, GFP is detected in all nonneuronal cells, perhaps explaining the refractoriness of neurons to systemic RNAi. To determine where SID-1 is localized within these cells, we examined the localization of a rescuing GFP-tagged SID-1 (SID1C::GFP). SID-1C::GFP rescued the systemic RNAi defect, suggesting that expression and localization would be representative of SID-1. SID-1C::GFP localized throughout cells with strong enrichment at the periphery of cells, consistent with plasma membrane association (Fig. 2B, upper panel). SID-1C::GFP was detected at a much reduced level compared to the promoter fusion construct and was detected in a reduced subset of cell types; however, sid-1 function is detected in cell types in which the fusion protein was not detected (e.g., body wall muscle). Curiously, SID-1C::GFP is most highly expressed in the cells and tissues that are directly exposed to the

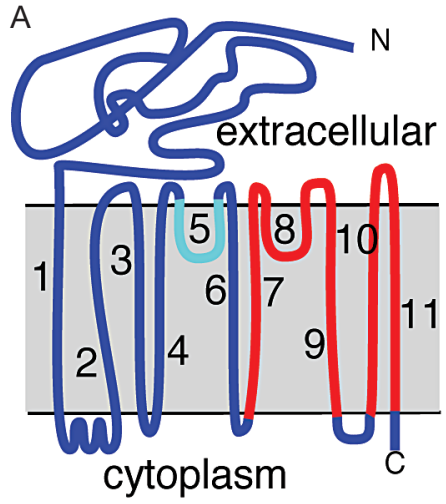

D

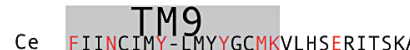

Ce FIINCIMY- 9 MYYGCMKVLHSERITSKAKLCGALSM10

Cb FILNCILY-LTYYALMKFASRESIELKAKVCGVSAIFGWLIAGFFFFQDDTDWTRTAAMSRALNTPCLLLDFFGSHDLWHMFGAIAGLFTFLFVSFVDDDLINTPKSSINMY Ce* FIGNLFIY-I IYYILMKMIYREKI PKRAIALLFAAVISWTCAGILFNQRVSDWSKMPAISRELNKPCIFLNFYDNHDLWHLSSAFAIFFSFTAINVIDDDLMFVMRNTIRVF Sp MIVNMVLY-MLFYLFMKGMCGENVLPSFLLL-VLTIALWGTAGSFFLKTNSGWQDSAARSREMNADCSIMGFYDAHDIWHLI SAFALFVSFVYLLLLDDNLDQTRQTDIPVF TC† LMGNSVLY-AVFYTGMKLVNGERICFEAI IYGLLAIAAWATATVYFLDNATLWTVTPAESROWNOECIVMSFYDKHDVWHLLSAPALYLTFMFLLSLDDDLVDTKREEITVF TC† FMGNTLLY-FSFYIVMKI INKERVNKL SLFFLSL SVLCAISAMYFFLNKSISWSRTPAQSROFNOECKLLRFYDFHDTWHFL SAIGMFFTFMVLLTLDDDLSHTHRNKTVVF Am LMSNLILYTTFYIIMICHKERILOPCIYIVLSIVFWAAYYFINKTISWELTSAQSRHYMKPCELHFFDSHDIWHFLALAMFSFMVLLTLDDDLIDVHRSQIPVF Am LMSNLIY MI HS FICNLLLY-LAFYIMKLRSSEKVLPVLFCIVATAVMAAALYFFFQNLSSWEGTPAESREKNRECILLDFFDDHDIWHFLSATALFF SFLVLLTLDDDLDDVRRDQIPVF Tn AICNLLLY-FAFYI IMKLRSGER IQCLALVCILFTAVWWGFALYFFFQGLSTWQKTPAESREHNRDCILLSFFDDHDVWHFLSSIAMFGSFLVLLTLDDDLDTVQRDKIFVF Dd FS-NLILYVLVRSNAMKI KYGEKVYLFVWVLFAIMFLSWGFGLYYFEISVTNKFYTFDESLLLNRPCI I FNYWDTHDLWHCNWNOKYFFIFLFLFF

Figure 2. SID-1 structure, function, localization, and conservation. (A) Topology of SID- 1 as determined by $\beta$-gal folding assay. The dark blue portions were demonstrated to be extracellular or intracellular constraining the orientation of the respective TM domains. The location and orientation of the light blue and red portions are undetermined. (B) Full-length functional SID-1::GFP (top panel) is enriched at the cell periphery (arrows) compared to GFP (bottom panel). Bar, $10 \mu \mathrm{m}$. (C) Injection of $g f p$ dsRNA into a single intestinal cell (arrowhead) in a sid-1 mutant animal that expresses nuclear-localized GFP in all cells demonstrates cell-autonomous RNAi. (D) Alignment of carboxy-terminal invertebrate and vertebrate SID-1 TM domains (gray). (Red) Amino acids that are identical among a majority of proteins; (blue) amino acids that are conserved among vertebrates. SID-1 homologs: (Ce) C. elegans; (Cb) C. briggsae; (Ce*) C. elegans ZK721.1; (Sp) Strongylocentrotus purpuratus; (Tc) Tribolium castaneum; (Am) Apis mellifera; (Mm) Mus musculus; (Hs) Homo sapiens; (Tn) Tetraodon nigroviridis; (Dd) Dictyostelium discoideum; (Tc ${ }^{\dagger}$ ) the two Tribolium homologs are similar but distinct. (Reprinted in part, with permission, from Feinberg and Hunter 2003 [@ AAAS].) 
environment: pharynx, intestine, sphincter muscles, excretory cell, and phasmids, plus the gonad, which is indirectly exposed to the environment via the vulva. The significance of this observation remains unknown, but it portends a role in the uptake of environmental molecules.

SID-1 homologs are readily detected in genome sequence databases (Fig. 2D). The most conserved region is the carboxy-terminal region, which contains the TM domains, although all homologs are predicted to contain a large extracellular domain and a similar number of TM domains. At least one and usually two SID-1 homologs are present in all sequenced vertebrate genomes. In invertebrates, SID-1 homologs are present in some but not all sequenced genomes. For example, Drosophila melanogaster, Anopheles gambiae (mosquito), and Ciona (sea squirt) do not appear to have SID-1 homologs, whereas clear homologs are readily detected in Apis mellifera (honeybee), Tribolium castaneum (flour beetle), Strongylocentrotus purpuratus (sea urchin), and Dictyostelium discoideum (slime mold). Systemic RNAi has been reported in honeybee and flour beetle (Bucher et al. 2002; Amdam et al. 2003). The possible function of SID-1 homologs is discussed below.

To characterize the function of $C$. elegans SID-1, RNAi targeting a variety of endogenous and reporter genes was initiated by tissue-specific transgenes expressing RNA hairpins, by injecting dsRNA into specific cells, tissues, and body compartments, and by feeding and soaking animals in dsRNA. By these assays, RNAi is effective in sid-1 mutants only within the cell(s) in which dsRNA is directly expressed or injected. For example, expression of $g f p$ hairpin RNA in the pharynx or body wall muscles silences GFP expression in those tissues only. Injection of $g f p$ dsRNA into the large injectionaccessible intestinal cells leads to silencing of GFP only in the injected cell (Fig. 2C). Similarly, injection directly into the syncytial germ line of dsRNA that targets maternally expressed genes results in highly penetrant phenotypes. However, silencing is never observed in adjacent cells or tissues and is not observed in the sid $-1^{-}$progeny of germ-line-injected mothers. These results are consistent with a requirement for sid-1 in the import or export of a silencing signal. To test the role of sid-1 in import of the silencing signal, we generated sid-1 genetic mosaics (animals composed of sid $-1^{+}$and sid $-1^{-}$cells) and scored RNAi silencing of GFP in individual muscle cells, which was initiated by feeding the animals bacteria expressing a $g f p$ hairpin. In all scored animals, GFP expression was silenced in sid- $1^{+}$cells and was not affected in any sid- $1^{-}$ cells (Fig. 3). This result shows that sid-1 is required cellautonomously for the import or processing of a silencing signal. In analogous experiments, a requirement for sid-1 for the export of a silencing signal has also been demonstrated (J. Shih and C.P. Hunter, unpubl.).

This phenotypic analysis is consistent with SID-1dependent uptake and export of the systemic silencing signal. To investigate the nature of this systemic silencing signal and the molecular mechanism of SID-1 activity, we developed a heterologous cell-based assay (Feinberg and Hunter 2003). Published reports indicated that Drosophila was capable of robust cell-autonomous RNAi but appeared

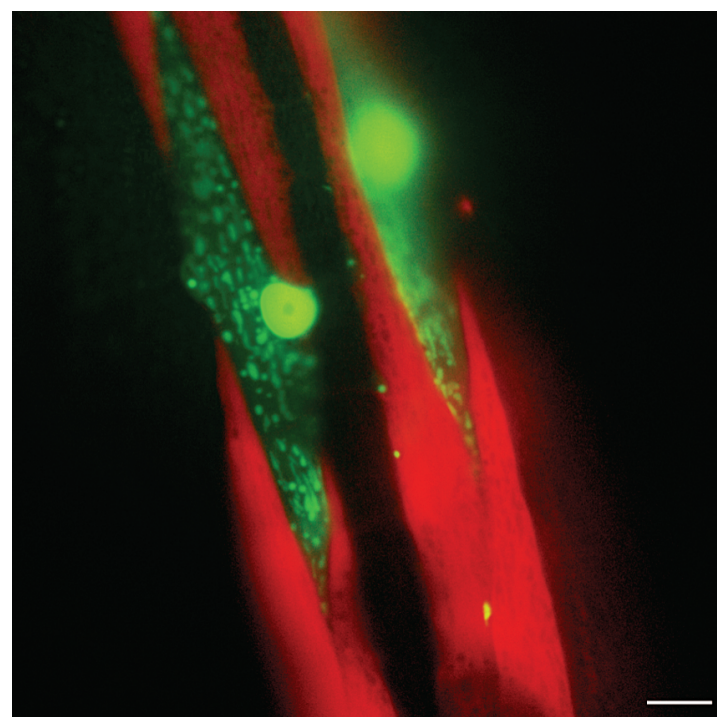

Figure 3. Mosaic analysis of sid-1 function. sid-1(+) body wall muscle cells are marked by the coexpression of DsRED2 (red). The two non-red muscle cells are resistant to bacteria-mediated RNAi and retain expression of GFP (green), whereas sid- $1(+)$ muscle cells (red) lose GFP expression. Bar, $10 \mu \mathrm{m}$. (Reprinted, with permission, from Winston et al. 2002 [@ AAAS].)

to mount at best inefficient systemic RNAi, perhaps consistent with the lack of a detectable SID-1 homolog in the fly genome. When briefly serum-starved and soaked in a high concentration of dsRNA, phagocytic Drosophila S2 cells can efficiently initiate RNAi via a dsRNA uptake mechanism that requires endocytosis (Ulvila et al. 2006). It is unknown how the dsRNA crosses the membrane to initiate RNAi. In this S2 cell system, we developed both a quantitative silencing assay targeting luciferase and a radiolabeled dsRNA uptake assay using transient transfection of either wild-type SID-1 (wt) or a missense allele SID-1 (qt2) as a negative control. In the luciferase-silencing assay, expression of SID-1(wt) enabled cells to mount an RNAi response in the presence of approximately $10^{5}$-fold less dsRNA than cells expressing the mutant SID-1 protein (Fig. 4A). This assay is quite sensitive, allowing us to detect silencing initiated at dsRNA concentrations below one dsRNA molecule per transfected cell. Using this assay, we determined that longer dsRNA was more effective at triggering sid-1-dependent silencing, either because it was taken up more efficiently or because it more efficiently interacted with the silencing machinery in the cell. The effective size cutoff is between 50 and 100 bp of dsRNA, with siRNAs effective only at the highest concentrations tested (Fig. 4B). This experiment suggested that systemic RNAi in the worm might be sensitive to the size of the trigger dsRNA. To address this possibility, we injected either a 50- or 100-bp dsRNA targeting the essential mex-3 gene directly into the syncytial germ line or the intestine. Injection of either dsRNA into the germ line yields high embryonic lethality, whereas injection into the intestine and requisite transport to the germ line is only effective with the 100-bp dsRNA. To confirm that this result does not merely reflect varying efficiency of the dsRNA sequences in initiating RNAi, we generated a 100-bp 
A
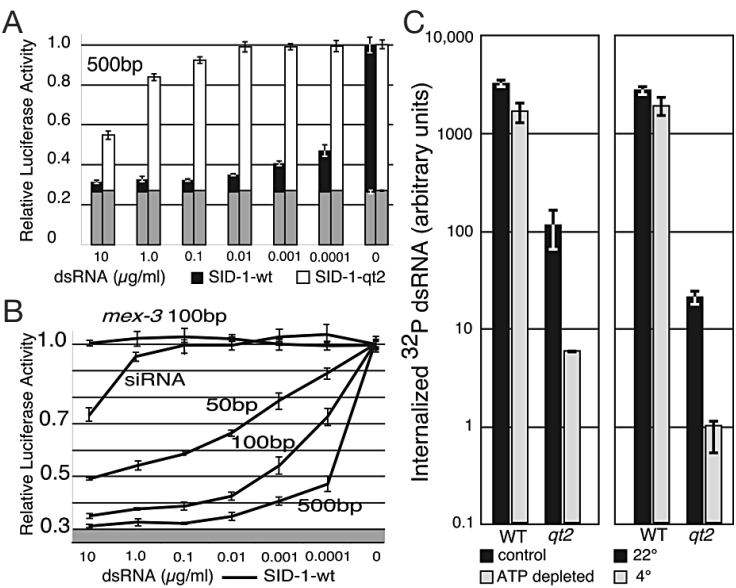

Figure 4. Characterization of SID-1 activity in Drosophila S2 cells. Wild-type (WT) or mutant (qt) SID-1 was transfected with a luciferase-expressing construct for activity assays. (A) Luciferase levels shown are relative to luciferase levels in cells exposed to no dsRNA. (Gray bars) Luciferase levels that remain at the end of the experiment in samples treated with the translation inhibitor cycloheximide $(50 \mu \mathrm{g} / \mathrm{ml})$ to control for protein stability. ( $B$ ) Summary of SID-1-WT data showing dsRNA sizedependent silencing. (C) SID-1 mediates passive uptake of dsRNA in S2 cells. SID-1-mediated dsRNA uptake is resistant to ATP depletion (left panel) and to reduced temperature (right panel). (Reprinted in part, with permission, from Feinberg and Hunter 2003 [@ AAAS.])

dsRNA containing the 50-bp mex-3 dsRNA fused to $50 \mathrm{bp}$ from luciferase. Intestinal injection of this dsRNA elicits efficient RNAi, confirming that dsRNA size, not merely sequence, determines systemic RNAi efficiency and explains the relatively poor systemic silencing capacity of shorter dsRNA (Parrish et al. 2000). Thus, the SID-1dependent silencing assay in $\mathrm{S} 2$ cells revealed specific properties of systemic RNAi in C. elegans.

To gain further mechanistic insight into SID-1 function, we modified the $\mathrm{S} 2$ cell system to demonstrate SID1-dependent intracellular accumulation of radiolabeled dsRNA and thereby showed that SID-1 is a dsRNA transporter (Fig. 4C) (Feinberg and Hunter 2003). In this assay, 100- and 500-bp dsRNAs accumulate in cells to at least fivefold higher levels than 50-bp dsRNA, consistent with the phenotypic effects observed in vitro and in vivo (M.C. Fitzgerald et al., unpubl.). Similar experiments have failed to detect the efficient transport of other nucleic acids, although this may reflect the lack of retention within cells. The ability of SID-1 to function in a heterologous system indicates that it either functions alone or accommodates Drosophila variants of protein cofactors. Remarkably, depleting ATP or performing uptake assays at $4^{\circ} \mathrm{C}$ has little effect on the extent or rate of uptake, arguing for a passive uptake mechanism most consistent with a function as a dsRNA channel (Fig. 4D). This presents a still-unsolved problem of how SID-1 activity is regulated, as a channel large enough to admit dsRNA could allow essential cellular components to exit the cell.

The wide phylogenetic conservation of SID-1 homologs and the demonstration of systemic RNAi in other invertebrates (Bucher et al. 2002; Amdam et al. 2003; Newmark et al. 2003; Turner et al. 2006) and in mammals (Soutschek et al. 2004) suggest that the function of SID-1 as a dsRNA transporter may be conserved and may even be the selected activity for this protein family. In fact, Duxbury et al. (2005) obtained evidence that supports this idea: These authors report that overexpressing a human SID-1 homolog in human cells enabled efficient passive siRNA uptake and silencing. Although other substrates were not tested, the human and C. elegans SID-1 proteins have similar activities. We eagerly await further evidence of dsRNA transport functions for vertebrate and invertebrate SID-1 homologs.

\section{SID-2}

The second largest complementation group recovered in the original screen identified SID-2, a single-pass transmembrane protein strongly expressed in the gut (Winston et al. 2002 and in prep.). This restricted expression pattern corresponds to the phenotype observed in sid-2 mutants, which are only defective for environmental RNAi, i.e., RNAi triggered by soaking in dsRNA or ingesting bacteria expressing dsRNA. Consequently, sid-2 mutants are fully sensitive to systemic RNAi triggered by transgene expression or microinjection into any tissues. Topology-determining experiments show that the predicted TM domain separates an extracellular aminoterminal domain from an intracellular carboxy-terminal domain. Fusing GFP to the intracellular carboxyl terminus of SID-2 (SID-2-C::GFP) produces a fusion gene that fully rescues the sid-2 environmental RNAi defect and strongly localizes GFP to intracellular and surfacelocalized membrane structures (Fig. 5).
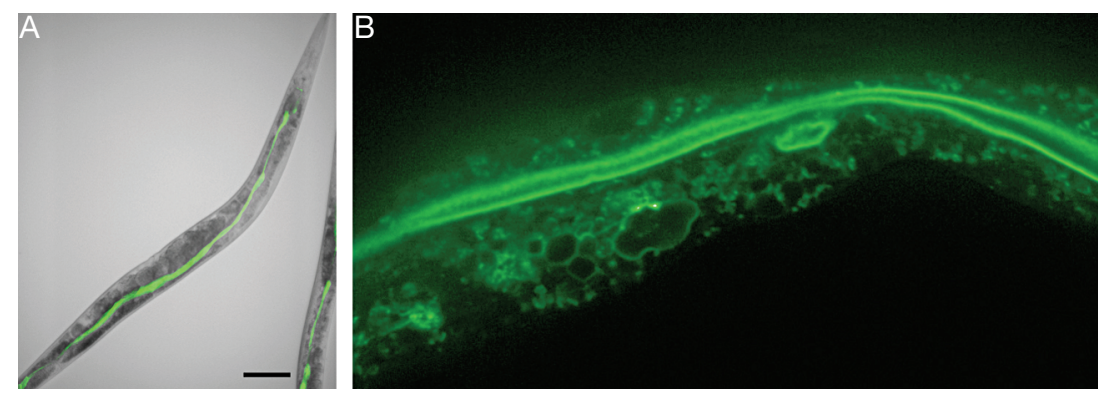

Figure 5. Localization of SID-2::GFP expression to intestinal lumen. (A) Fluorescent and white light overlay at low magnification and $(B)$ confocal section at high magnification. (Confocal image courtesy of Daniel Schott.) 
In contrast to the wide phylogenetic distribution of sid-1, the only readily detected sid-2 homologous genes are from Caenorhabditis briggsae and C. remanei. The degree of amino acid sequence conservation among the three domains of SID-2 from all three species is similar: 23\% amino-terminal (190 amino acids), 86\% TM (21 amino acids), and 53\% carboxy-terminal (100 amino acids), despite the relative recent divergence of $C$. briggsae and $C$. remanei. Among these three nematodes, only C. elegans efficiently initiates RNAi in response to environmental exposure to dsRNA. Given the relative lack of sequence conservation in the amino-terminal domain of SID-2, we suspected that C. elegans had gained this activity or C. briggsae and C. remanei had lost this activity. A Cb-SID-2::GFP fusion protein expressed in $C$. briggsae showed intestinal expression localized to the luminal membrane, indicating that changes in gene expression and protein localization do not account for the different sensitivities to environmental dsRNA. Furthermore, expression of the Cb-SID-2::GFP in C. elegans failed to rescue the sid-2 mutant phenotype; conversely, expression of Ce-SID-2-C::GFP did confer environmental RNAi sensitivity on C. briggsae. To address the possibility that the amino acid changes in the highly divergent extracellular domain underlie the differential response to environmental dsRNA, we fused the Ce-SID-2 extracellular and TM domains to the Cb-SID-2 intracellular domain fused to GFP. This construct rescued the C. elegans sid-2 mutant, thus localizing the relevant activity to the extracellular/TM domains. Further analysis of these domains is in progress.

To determine whether environmental RNAi sensitivity arose in C. elegans or had been lost in C. briggsae and $C$. remanei, we undertook a broad analysis of systemic RNAi among extant Caenorhabditis species (Fig. 6). We

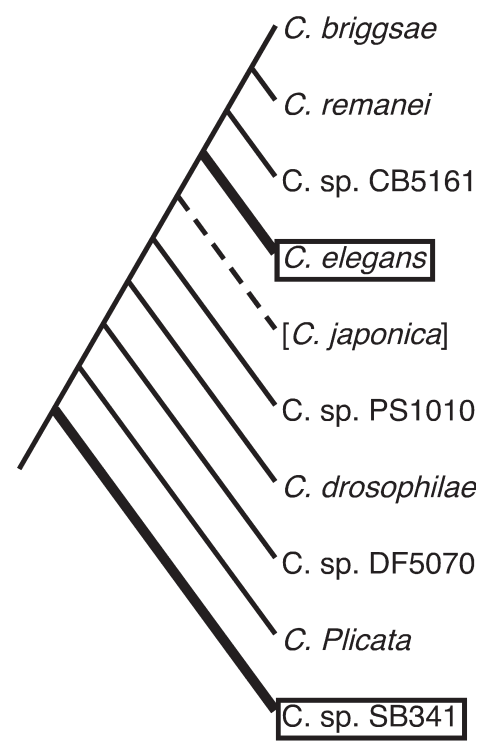

Figure 6. Phylogenetic relationship of Caenorhabditis species (Kiontke et al. 2004) and systemic and environmental RNAi proficiency. C. japonica (dashed line) was not tested. Only C. sp. CB5161 was deficient for systemic RNAi, whereas only $C$. elegans and C. sp. SB341 (boxed) were proficient at environmental RNAi. produced a set of species-specific RNA polymerase II subunit dsRNA that produced a similar early embryonic arrest phenotype among the progeny embryos (cell cycle arrest around gastrulation) when injected into the gonad of each species, showing that the dsRNA effectively initiated RNAi in each species. To test for systemic RNAi, we measured embryonic lethality among the progeny of intestine-injected mothers, and to test for environmental RNAi, we measured embryonic lethality among the progeny of mothers soaked in dsRNA overnight. All but one species was sensitive to intestine-injected dsRNA, showing that systemic RNAi is broadly conserved among Caenorhabditis. It will be interesting to learn whether sid-1 has been conserved in this systemic RNAi-defective species. In contrast, only $C$. elegans and one distantly related and unnamed species were sensitive to environmental RNAi. This result indicates that sensitivity to environmental RNAi is rare and is not a general property of nematodes. Thus, this property of SID-2 likely arose within C. elegans. Ongoing investigations into the natural ecology of Caenorhabditis species may suggest a role, for example, in acquiring sequence-specific resistance to pathogens, for such an activity (Fitch 2005; Hong and Sommer 2006).

\section{CONCLUSIONS AND PROSPECTUS}

The initial genetic screen identified three large complementation groups with strong effects on systemic RNAi. Two of these, sid-1 and sid-2, represent two easily mutated and recoverable mutants that disrupt the uptake and distribution of dsRNA in C. elegans. All other recovered alleles, which represent at least five additional genes, were recovered at $1-10 \%$ of the frequency of sid -1 . Thus, new screens designed to avoid or eliminate the recovery of sid-1 and sid-2 alleles should identify new genes and pathways that function with sid-1 or sid-2. The identification of genes that regulate the activity of SID-1 and SID-2 may provide an insight into the function of these pathways. For example, perhaps the SID proteins provide an antiviral defense similar to the role of systemic silencing in plants (Baulcombe 2004). Although data supporting an antiviral role for systemic RNAi have not been reported, it is clear that RNAi is antiviral in C. elegans (Lu et al. 2005; Schott et al. 2005; Wilkens et al. 2005). Alternatively, microRNAs or mRNAs may be generally or selectively transported between cells, tissues, or animals. Finally, it should not be assumed that dsRNA is the only substrate: A channel large enough to permit passage of long dsRNA through a membrane could accommodate many different large molecules.

The SID-1 protein family is ancient, with SID-1 homologs present in Dictyostelium, many invertebrates, and all sequenced vertebrates. However, it is unknown whether any of these homologs function in dsRNA transport. In C. elegans, ZK721.1 encodes a protein slightly more similar to the vertebrate SID-1 homologs than SID-1 itself. However, deletion of ZK721.1 does not have a detectable systemic RNAi defect in any tested $C$. elegans tissue. In contrast, overexpression of a human SID-1 homolog enables efficient RNAi in human cells. This hints 
that the evolutionarily selected function for some of the SID-1 homologs is indeed dsRNA transport. Investigation of the functions and substrate selectivity of these proteins will be important for the development of effective therapeutic applications of RNAi to human health.

\section{ACKNOWLEDGMENTS}

We thank Antony Jose and Daniel Schott for comments on the manuscript. This project was initiated with funds provided by the Beckman Young Investigator Foundation and continues with the support of the National Science Foundation (MCB-0417102) and the National Institutes of Health (GM06989).

\section{REFERENCES}

Amdam G.V., Simoes Z.L., Guidugli K.R., Norberg K., and Omholt S.W. 2003. Disruption of vitellogenin gene function in adult honeybees by intra-abdominal injection of doublestranded RNA. BMC Biotechnol. 3: 1.

Baulcombe D. 2004. RNA silencing in plants. Nature 431: 356.

Bucher G., Scholten J., and Klingler M. 2002. Parental RNAi in Tribolium (Coleoptera). Curr. Biol. 12: R85.

Duxbury M.S., Ashley S.W., and Whang E.E. 2005. RNA interference: A mammalian SID-1 homologue enhances siRNA uptake and gene silencing efficacy in human cells. Biochem. Biophys. Res. Commun. 331: 459.

Feinberg E.H. and Hunter C.P. 2003. Transport of dsRNA into cells by the transmembrane protein SID-1. Science 301: 1545.

Fire A., Xu S., Montgomery M.K., Kostas S.A., Driver S.E., and Mello C.C. 1998. Potent and specific genetic interference by double-stranded RNA in Caenorhabditis elegans. Nature 391: 806.

Fitch D.H. 2005. Evolution: An ecological context for C. elegans. Curr. Biol. 15: R655.

Hong R.L. and Sommer R.J. 2006. Pristionchus pacificus: A well-rounded nematode. Bioessays 28: 651.

Kiontke K., Gavin N.P., Raynes Y., Roehrig C., Piano F., and Fitch D.H. 2004. Caenorhabditis phylogeny predicts convergence of hermaphroditism and extensive intron loss. Proc. Natl. Acad. Sci. 101: 9003.

Lu R., Maduro M., Li F., Li H.W., Broitman-Maduro G., Li W.X., and Ding S.W. 2005. Animal virus replication and RNAi-mediated antiviral silencing in Caenorhabditis elegans. Nature 436: 1040.
Newmark P.A., Reddien P.W., Cebria F., and Sanchez Alvarado A. 2003. Ingestion of bacterially expressed double-stranded RNA inhibits gene expression in planarians. Proc. Natl. Acad. Sci. (suppl. 1) 100: 11861.

Parrish S., Fleenor J., Xu S., Mello C., and Fire A. 2000. Functional anatomy of a dsRNA trigger: Differential requirement for the two trigger strands in RNA interference. Mol. Cell 6: 1077.

Schott D.H., Cureton D.K., Whelan S.P., and Hunter C.P. 2005. An antiviral role for the RNA interference machinery in Caenorhabditis elegans. Proc. Natl. Acad. Sci. 102: 18420.

Soutschek J., Akinc A., Bramlage B., Charisse K., Constien R., Donoghue M., Elbashir S., Geick A., Hadwiger P., Harborth J., et al. 2004. Therapeutic silencing of an endogenous gene by systemic administration of modified siRNAs. Nature 432: 173.

Tabara H., Grishok A., and Mello C.C. 1998. RNAi in C. elegans: Soaking in the genome sequence. Science 282: 430.

Tabara H., Sarkissian M., Kelly W.G., Fleenor J., Grishok A., Timmons L., Fire A., and Mello C.C. 1999. The $r d e-1$ gene, RNA interference, and transposon silencing in C. elegans. Cell 99: 123.

Tijsterman M., May R.C., Simmer F., Okihara K.L., and Plasterk R.H. 2004. Genes required for systemic RNA interference in Caenorhabditis elegans. Curr. Biol. 14: 111.

Timmons L. and Fire A. 1998. Specific interference by ingested dsRNA. Nature 395: 854.

Timmons L., Tabara H., Mello C.C., and Fire A.Z. 2003. Inducible systemic RNA silencing in Caenorhabditis elegans. Mol. Biol. Cell 14: 2972.

Turner C.T., Davy M.W., Macdiarmid R.M., Plummer K.M., Birch N.P., and Newcomb R.D. 2006. RNA interference in the light brown apple moth, Epiphyas postvittana (Walker) induced by double-stranded RNA feeding. Insect Mol. Biol. 15: 383 .

Ulvila J., Parikka M., Kleino A., Sormunen R., Ezekowitz R.A., Kocks C., and Ramet M. 2006. Double-stranded RNA is internalized by scavenger receptor-mediated endocytosis in Drosophila S2 cells. J. Biol. Chem. 281: 14370.

Wilkins C., Dishongh R., Moore S.C., Whitt M.A., Chow M., and Machaca K. 2005. RNA interference is an antiviral defence mechanism in Caenorhabditis elegans. Nature 436: 1044.

Winston W.M., Molodowitch C., and Hunter C.P. 2002. Systemic RNAi in C. elegans requires the putative transmembrane protein SID-1. Science 295: 2456.

Zimmermann T.S., Lee A.C., Akinc A., Bramlage B., Bumcrot D., Fedoruk M.N., Harborth J., Heyes J.A., Jeffs L.B., John M., et al. 2006. RNAi-mediated gene silencing in non-human primates. Nature 441: 111. 


\section{$8_{\mathrm{CSH}}^{\infty} \mathrm{\&}$ Cold Spring Harbor Symposia SYMPOSIA}

\section{Systemic RNAi in Caenorhabditis elegans}

C.P. HUNTER, W.M. WINSTON, C. MOLODOWITCH, et al.

Cold Spring Harb Symp Quant Biol 2006 71: 95-100

Access the most recent version at doi:10.1101/sqb.2006.71.060

References This article cites 24 articles, 8 of which can be accessed free at: http://symposium.cshlp.org/content/71/95.full.html\#ref-list-1

\section{License}

Email Alerting Receive free email alerts when new articles cite this article - sign up in Service the box at the top right corner of the article or click here. 